\title{
Genetic and biochemical identification of the chorismate mutase from Corynebacterium glutamicum
}

Correspondence

Shuang-Jiang Liu

liusj@sun.im.ac.cn

Received 5 April 2009

Revised 28 June 2009

Accepted 3 July 2009
Pan-Pan Li, ${ }^{1} \dagger$ Ya-Jun Liu ${ }^{1} \dagger$ and Shuang-Jiang Liu ${ }^{1,2}$

\author{
${ }^{1}$ State Key Laboratory of Microbial Resources, Institute of Microbiology, Chinese Academy of \\ Sciences, Beijing 100101, PR China \\ ${ }^{2}$ Environmental Microbiology and Biotechnology Research Center, Institute of Microbiology, \\ Chinese Academy of Sciences, Beijing 100101, PR China
}

\section{INTRODUCTION}

Chorismate mutases (CMs, EC 5.4.99.5) are of interest for bioindustry and medicine, because the enzyme is the target to improve production of aromatic amino acids such as Lphenylalanine (Phe) and L-tyrosine (Tyr) (Chavez-Bejar et al., 2008; Yakandawala et al., 2008) as well as being a putative new drug target against deadly diseases such as tuberculosis (Qamra et al., 2006; Schneider et al., 2008). CMs catalyse the conversion of chorismate to prephenate, a reaction also known as the Claisen rearrangement, and the only naturally occurring Claisen reaction in the primary metabolism of living organisms (Krappmann et al., 1999; Sugimoto \& Shiio, 1980a). This conversion is a key step in the biosynthetic pathway of Phe and Tyr in archaea, bacteria, fungi and plants (Helmstaedt et al., 2004).

TThese authors contributed equally to this work.

Abbreviations: $\mathrm{CD}$, circular dichroism; $\mathrm{CM}$, chorismate mutase; DAHP, 3-deoxy-D-arabino-heptulosonate 7-phosphate.
Examples of CMs that have been characterized are the EcCM from Escherichia coli (Hudson et al., 1984), the MjCM from Methanococcus jannaschii (MacBeath et al., 1998), the ScCM from Saccharomyces cerevisiae (Ball et al., 1986), the HpCM from Hansenula polymorpha (Krappmann et al., 2000), the MtbCM from Mycobacterium tuberculosis (Prakash et al., 2005), the AtCM from Arabidopsis thaliana (Eberhard et al., 1993), the BsCM from Bacillus subtilis (Gray et al., 1990), and the TtCM from Thermus thermophilus (Helmstaedt et al., 2004). Although those CMs are structurally conserved (Lee et al., 1995; Chook et al., 1993; Strater et al., 1997), their amino acid sequences are much less similar. For example, the secreted MtbCM (encoded by Rv1885c) shares only 10$20 \%$ identity with EcCM and ScCM (Qamra et al., 2006). Thus, identification of $\mathrm{CM}$ genes based on sequence homology from genomes is problematic. Prokaryotic CMs are grouped into either the AroQ class or the AroH class based on their crystal structure information (Chook et al., 1994; Strater et al., 1997; Calhoun et al., 2001). The 
AroQ class CMs are dimeric and folded as a helix bundle. The AroH class is trimeric and folded as a pseudo- $\alpha / \beta$ barrel (Helmstaedt et al., 2001). The AroQ class CMs are further divided into cytoplasmic and monofunctional CMs that are not subject to allosteric regulation [e.g. Rv0948c from Mycobacterium tuberculosis (Schneider et al., 2008)], periplasmic/secreted and monofunctional CMs [e.g. Rv1885c from M. tuberculosis (Prakash et al., 2005)], and multiple functional fusion CMs [e.g. chorismate mutase/ prephenate dehydrogenase from E. coli (Hudson et al., 1984)].

Corynebacterium glutamicum is commercially used for the production of amino acids, including the aromatic amino acids Phe and Tyr (Ikeda, 2005). Because of low sequence similarity among the CMs, CM was not annotated in the initial genome sequence of C. glutamicum (Ikeda \& Nakagawa, 2003). Later on, Ikeda $(2005,2006)$ reported ncgl0819, which had been annotated as a 'conserved hypothetical protein', to be a putative $\mathrm{CM}$ gene. But experimental identification of this putative CM gene was not carried out. In this study, we verified experimentally that $n c g l 0819$ is the sole gene encoding an active CM in C. glutamicum (CM0819). CM0819 was shown to belong to the cytoplasmic and monofunctional CMs of the AroQ class and is not subject to feedback inhibition by aromatic amino acids (Phe, Trp, Tyr). Two 3-deoxy-D-arabinoheptulosonate 7-phosphate (DAHP) synthases (DS0950 and DS2098, formerly NCgl0950 and NCgl2098) had been previously identified from C. glutamicum. CM0819 interacted with DS2098 from C. glutamicum and this interaction resulted in allosteric regulation of the DAHP synthase activity.

\section{METHODS}

Bacterial strains, plasmids and culture conditions. Bacterial strains and plasmids used in this study are listed in Table 1. E. coli strains were grown aerobically on a rotary shaker (150 r.p.m.) at $37{ }^{\circ} \mathrm{C}$ in Luria-Bertani (LB) broth or on LB plates with $1.5 \%(\mathrm{w} / \mathrm{v})$ agar. C. glutamicum strains were routinely grown at $30{ }^{\circ} \mathrm{C}$ in $\mathrm{LB}$ medium or in mineral salts medium (Konopka, 1993), which was adjusted to $\mathrm{pH} 7.5$ and supplemented with biotin $\left(100 \mu \mathrm{g} \mathrm{l}^{-1}\right)$ and thiamine $\left(500 \mu \mathrm{g} \mathrm{l}^{-1}\right)$ instead of yeast extract, on a rotary shaker (150 r.p.m.). When needed, glucose and aromatic amino acids (Phe, Tyr, Trp) were added as carbon and energy sources at final concentrations of $2 \mathrm{mM}$ and $0.2 \mathrm{mM}$, respectively. Cellular growth was monitored by measuring the turbidity at $600 \mathrm{~nm}$ using a spectrophotometer. For generation of mutants and maintenance of $C$. glutamicum, BHIS (brain heart broth with $0.5 \mathrm{M}$ sorbitol) medium was used. Antibiotics were used at the following concentrations: kanamycin, $50 \mu \mathrm{g} \mathrm{ml}^{-1}$ for E. coli and $25 \mu \mathrm{g} \mathrm{ml}^{-1}$ for C. glutamicum; ampicillin, $100 \mu \mathrm{g} \mathrm{ml}^{-1}$ for E. coli; chloramphenicol, $20 \mu \mathrm{g} \mathrm{ml}^{-1}$ for E. coli and $10 \mu \mathrm{g} \mathrm{ml}^{-1}$ for C. glutamicum; nalidixic acid, $100 \mu \mathrm{g} \mathrm{ml}^{-1}$ for C. glutamicum.

DNA and protein sequences analysis. The genome sequence of $C$. glutamicum ATCC 13032 (accession no. 003450) was retrieved from GenBank. Sequence comparisons and database searches were carried out using the BLAST service of the NCBI website (http:// www.ncbi.nlm.nih.gov). The protein secondary structure prediction was carried out with online protein structure prediction servers, namely YASPIN (http://ibi.vu.nl/programs/), SABLE (http://sable.cchmc.org/), PORTER (http://distill.ucd.ie/porter/), GARNIER (http:// vm-bioinfo.toulouse.inra.fr/emboss/emboss.cgi/garnier), QL server (http://abs.cit.nih.gov/ql1) and PROF (http://www.predictprotein. $\operatorname{org} /)$.

DNA extraction and manipulation. The total genomic DNA of $C$. glutamicum was isolated according to the procedure of Tauch et al. (1995). DNA restriction enzyme digestion, plasmid isolation and agarose gel electrophoresis were carried out as described by Sambrook et al. (1989). E. coli and C. glutamicum were transformed by electroporation according to the method of Tauch et al. (2002).

Amplification of DNA fragments with PCR and construction of plasmids. PCRs were performed by using Pfu DNA polymerase or Taq DNA polymerase (Transgen). PCR products were purified using an agarose gel DNA fragment recovery kit (TIANGEN). Cloning of PCR fragments was performed with the pEASY-T1 cloning vector systems (Transgen). Various plasmids (Table 1) for genetic disruption and complementation in C. glutamicum and for expression in E. coli were constructed based on pK18mobsacB, pXMJ19, pET21a and pET28a. Primers used for amplification of the entire target gene or target fragment are listed in Table 1. Gene deletion was performed by gene splicing through an overlap extension method called Gene Soeing (Horton et al., 1989). According to the genome sequence of $C$. glutamicum, fragments of about $300 \mathrm{bp}$ from both sides of ncgl0819 (homologous arms) were amplified and ligated together to generate the disrupted gene $\Delta n c g l 0819$. Then $\Delta n c g l 0819$ was fused into $\mathrm{pK} 18$ mobsacB to generate plasmid pK18mobsacB- $\Delta$ ncgl0819. For complementation, pXMJ19-ncgl0819 was constructed by insertion of PCR-amplified intact gene ncglo819 into pXMJ19 (Jakoby et al., 1999). A plasmid for heterologous expression of the target gene was created by ligation of the PCR-amplified gene into pET28a and pET21a.

Genetic disruption and complementation in C. glutamicum. The pK18mobsacB derivative was transformed into C. glutamicum RES167 by electroporation (Tauch et al., 2002) to replace the target gene ncgl0819 with $\Delta$ ncgl0819 by homologous recombination. Screening for the first and second recombination events, as well as confirmation of the chromosomal deletion, was performed as described previously (Schafer et al., 1994). The resulting strain was designated C. glutamicum RES167 $n$ ngl0819 (Table 1). The deletion of the target gene $n c g l 0819$ in the pK18mobsacB derivative and in the C. glutamicum mutant was verified by PCR amplification and DNA sequencing. Complementary plasmid pXMJ19-ncgl0819 was introduced into C. glutamicum RES167Ancgl0819 by electroporation. Expression of ncglo819 in C. glutamicum was induced by the direct addition of IPTG to the culture, to a final concentration of $0.6 \mathrm{mM}$.

Heterologous expression in E. coli, preparation of cellular Iysate, and protein purification. Plasmids pET28-ncgl0950, pET28ncgl2098, pET28-ncgl0819 and pET21-ncgl2098 were transformed into E. coli BL21(DE3) by electroporation for heterologous expression of the corresponding genes (ncgl0950, ncgl2098, ncgl0819). Synthesis of recombinant proteins in E. coli BL21(DE3) cells was initiated by the addition of IPTG $(0.6 \mathrm{mM})$, and cultivation was continued for an additional $10 \mathrm{~h}$ at $14{ }^{\circ} \mathrm{C}$. Cells were harvested by centrifugation at

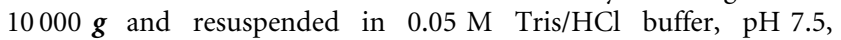
containing $1 \mathrm{mM}$ DTT (Ikeda \& Nakagawa, 2003). For DS0950 and DS2098, cells were washed twice with $0.2 \% \mathrm{KCl}$ before resuspension. Then cells were disrupted by sonification in an ice-water bath. Cellular lysate was centrifuged at $20000 \mathrm{~g}$ for $30 \mathrm{~min}$ at $4{ }^{\circ} \mathrm{C}$ and the supernatant was used for protein purification. The hexahistidine cascade of pET28a was fused to the target genes at the $5^{\prime}$ end during plasmid construction. Then the recombinant proteins were purified 
Table 1. Bacterial strains, plasmids and primers used

\begin{tabular}{|c|c|c|}
\hline Strain/plasmid/primer & Relevant characteristics/sequences & Source/reference/notes \\
\hline \multicolumn{3}{|l|}{ E. coli } \\
\hline XL1-Blue & $\begin{array}{l}\text { supE44 hsdR17 recA1 endA1 gyrA46 thi relA1 lac } \mathrm{F}^{\prime}\left[\text { proAB }{ }^{+} \text {lacl }{ }^{\mathrm{q}} \text { lacZLM15 }\right. \\
\left.\operatorname{Tn} 10\left(\operatorname{Tet}^{\mathrm{R}}\right)\right]\end{array}$ & Stratagene (Cat. No. 200249) \\
\hline BL21(DE3) & hsdS gal ( $\lambda$ cIts857 ind-l Sam7 nin-5 lacUV5-T7 gene 1) & Novagen (Cat. No. 69450-3) \\
\hline \multicolumn{3}{|l|}{ C. glutamicum } \\
\hline RES167 & Restriction-deficient mutant of ATCC 13032, $\Delta($ cglIM-cglIR-cglIIR $)$ & Gift from University of Bielefeld \\
\hline 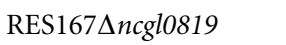 & DNA fragment encoding amino acids $1-96$ of ncgl0819 deleted & This study \\
\hline \multicolumn{3}{|c|}{ 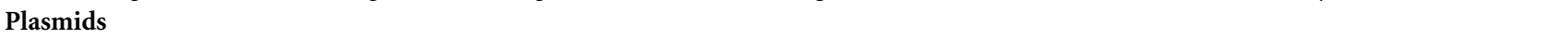 } \\
\hline pK18mobsacB & Mobilizable vector, allows for selection of double crossover in C. glutamicum & Schafer et al. (1994) \\
\hline $\begin{array}{l}\text { pK18mobsacB- } \\
\Delta n c g l 0819\end{array}$ & 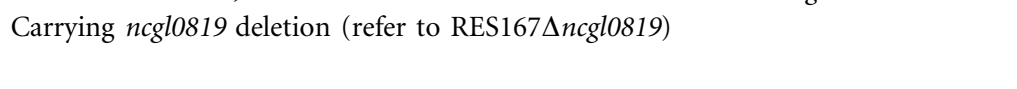 & This study \\
\hline pXMJ19 & Shuttle vector $\left(\mathrm{Cam}^{\mathrm{r}}, \mathrm{Ptac}\right.$, lacl $^{q}, \mathrm{pBL1}$ ori $V_{\text {C. glu }} \mathrm{pK} 18$ ori $\left.V_{\text {E. coli }}\right)$ & Jakoby et al. (1999) \\
\hline pXMJ19-ncglo819 & Carrying $n c g l 0819$ (to generate complementation for $n c g l 0819$ ) & This study \\
\hline pET28 & Expression vector with $\mathrm{N}$-terminal $\mathrm{His}_{6}$ affinity tag & Novagen \\
\hline pET28-ncgl0950 & pET28a derivative for expression of $n c g l 0950$ & Liu et al. (2008) \\
\hline pET28-ncgl2098 & pET28a derivative for expression of $n c g l 2098$ & Liu et al. (2008) \\
\hline pET28-ncgl0819 & pET28a derivative for expression of $n c g l 0819$ & This study \\
\hline pET21 & Expression vector with no $\mathrm{His}_{6}$ affinity tag & Novagen \\
\hline pET21-ncgl2098 & pET21a derivative for expression of ncgl2098 & This study \\
\hline \multicolumn{3}{|l|}{ Primers ${ }^{*}$} \\
\hline 0819a & GACGGATCCGACACGCTCACGCATCTC $($ BamHI) & $\begin{array}{l}\text { To generate pK18mobsacB- } \\
\Delta n c g l 0819\end{array}$ \\
\hline $0819 b$ & CTTATCCGAGTTTTCCGC GCACACTATTCTGCCACG & \\
\hline $0819 c$ & GCGGAAAACTCGGATAAG & \\
\hline 0819d & CGAATTCTAGACCTGTGACGATGGC (EcoRI) & \\
\hline 0819F & GACGGATCCAAAGGAGGAGACCATATGACTAATGCAGGTGAC (BamHI/NdeI) & $\begin{array}{l}\text { To generate pXMJ19-ncgl0819 } \\
\text { and pET28-ncgl0819 }\end{array}$ \\
\hline 0819R & CGAATTCACTTATCCGAGTTTTCCG (EcoRI) & \\
\hline
\end{tabular}

${ }^{\star}$ Restriction enzyme sites are underlined. Ribosome-binding sites are in bold type. Letters in italic denote the annealing regions for Gene Soeing PCR.

with the His-Bind protein purification kit (Novagen) according to the manufacturer's instructions.

Interaction between CM0819 and DS2098. In order to determine whether CM0819 and DS2098 interact with each other, recombinant E. coli cellular lysates containing CM0819 or DS2098 were sequentially loaded onto a His-Bind protein purification column (Novagen). The E. coli BL21(DE3)/pET28-ncglo819 cellular lysate was first loaded, and the column was then washed to remove residue proteins. CM0819 expressed in E. coli BL21(DE3)/pET28- $n$ cgl0819 has a His-tag, so it bound to the resin and remained within the column after washing with buffer. The BL21(DE3)/pET21-ncgl2098 cellular lysate was then loaded. DS2098 had no His-tag, so it was not able to bind with resin and would be easily washed out with buffer. However, if CM0819 interacted with DS2098 and the two formed a complex, this DS2098 would remain within the column. Only when CM0819 was eluted with eluant was DS2098 eluted simultaneously with CM0819. As controls, in parallel, cellular lysates of BL21(DE3)/ pET28-ncgl0819, BL21(DE3)/pET21-ncgl2098, or BL21(DE3)/pET28ncgl2919 (carrying fumarylpyruvate hydrolase) (Shen et al., 2005) were loaded onto the same His-Bind protein purification columns.

Assay of CM. CM activity was assayed following the procedure of Barrie and Graham (Davidson \& Hudson, 1987). The assay mixture contained $50 \mathrm{mM}$ Tris/ $\mathrm{HCl}$ buffer, $\mathrm{pH} 7.5$ (containing $1 \mathrm{mM}$ DTT), and $1 \mathrm{mM}$ chorismic acid in a total volume of $0.2 \mathrm{ml}$. The mixture was incubated at $37{ }^{\circ} \mathrm{C}$ for $5 \mathrm{~min}$ and then enzyme was added to initiate the reaction; $10 \mathrm{~min}$ later the reaction was stopped by addition of $0.4 \mathrm{ml} 1 \mathrm{M} \mathrm{HCl}$. After a further $10 \mathrm{~min}$ at $37{ }^{\circ} \mathrm{C}$ to convert prephenate to phenylpyruvate, $0.8 \mathrm{ml} 2.5 \mathrm{M} \mathrm{NaOH}$ was added and the absorbance at $320 \mathrm{~nm}$ of the final solution was measured immediately. A blank correction was made using a solution treated as above, except that the enzyme was added after the $\mathrm{NaOH}$. One unit of activity of CM is defined as the amount of enzyme that catalyses the conversion of $1 \mu \mathrm{mol}$ chorismate to prephenate per min at $37^{\circ} \mathrm{C}$; the absorption coefficient of phenylpyruvate at $320 \mathrm{~nm}$ $\left(\varepsilon_{320}\right)$ was determined as $17.5 \times 10^{3} \mathrm{M}^{-1} \mathrm{~cm}^{-1}$ (Davidson \& Hudson, 1987). Aromatic amino acids $(1 \mathrm{mM})$ were added to the mixture when feedback inhibition of the enzyme was tested.

$K_{\mathrm{m}}, V_{\max }$ and $k_{\text {cat }}$ values were obtained by fitting the kinetic data directly to the equation $v=V_{\max } \times S /\left(K_{\mathrm{m}}+S\right)$, where $v$ is the initial velocity, $V_{\max }$ is the maximal initial velocity, $K_{\mathrm{m}}$ is the Michaelis constant, and $S$ is the variable substrate concentration.

Assay of DAHP synthase. DAHP synthase activity was determined according to Siehl (1997). The assay mixture contained $50 \mathrm{mM}$ Tris/ $\mathrm{HCl}$ buffer, $\mathrm{pH} 7.5,5 \mathrm{mM}$ phosphoenolpyruvate, $2 \mathrm{mM}$ E4P (erythrose 4-phosphate) and enzyme in a volume of $75 \mu$ l. The mixture was incubated at $30{ }^{\circ} \mathrm{C}$ for $10 \mathrm{~min}$. The rate of reaction was proportional to the enzyme concentration and was linear with time. The reaction was initiated by the addition of E4P or enzyme and 
terminated by the addition of $400 \mu 110 \%(\mathrm{w} / \mathrm{v})$ TCA. Then $100 \mu \mathrm{l}$ $25 \mathrm{mM} \mathrm{NaIO}_{4}$ in $62.5 \mathrm{mM} \mathrm{H}_{2} \mathrm{SO}_{4}$ was added to the mixture and incubated at $37{ }^{\circ} \mathrm{C}$ for $30 \mathrm{~min}$, after which the reaction was stopped by addition of $100 \mu \mathrm{l} 2 \%(\mathrm{w} / \mathrm{v}) \mathrm{Na}_{2} \mathrm{SO}_{3}$ in $0.5 \mathrm{M} \mathrm{HCl}$, and the tubes were mixed before adding $1 \mathrm{ml}$ of $0.36 \%(\mathrm{w} / \mathrm{v})$ thiobarbituric acid. Then the tubes were incubated at $100{ }^{\circ} \mathrm{C}$ for $10 \mathrm{~min}$ and the colour of the mixture turned to pink. The tubes were allowed to cool to room temperature and the absorbance at $549 \mathrm{~nm}$ was measured. Reaction mixtures without enzyme were run in parallel and used as controls. One unit of activity is defined as the amount of enzyme that catalyses the synthesis of $1 \mu \mathrm{mol}$ DAHP per min at $30{ }^{\circ} \mathrm{C}$. For calculation, an absorption coefficient of DAHP at $549 \mathrm{~nm}\left(\varepsilon_{549}\right)$ of $4.5 \times 10^{4} \mathrm{M}^{-1}$ $\mathrm{cm}^{-1}$ was adopted (Schoner \& Herrmann, 1976).

Inhibition to DAHP synthase activity by chorismate or prephenate was determined by inclusion of chorismate or prephenate in the assay mixtures. Parallel experiments without enzymes in the assay mixture were run as control.

SDS-PAGE, determinations of molecular mass of the purified enzymes and protein estimation. SDS-PAGE was conducted with $5 \%$ stacking gels and $12 \%$ resolving gel and was run with a MiniPROTEAN II Electrophoresis Cell (Bio-Rad) according to the manufacturer's instructions. After electrophoresis, the protein bands were visualized by Coomassie brilliant blue staining. Apparent molecular mass was estimated according to the relative mobility of protein markers (Transgen). The native molecular mass of the enzyme was estimated by gel filtration chromatography on a prepacked Superdex 200HR column (Pharmacia). The column was equilibrated and eluted with $50 \mathrm{mM}$ Tris/ $\mathrm{HCl}(\mathrm{pH}$ 7.5) containing

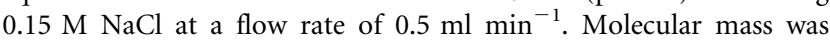
calculated according to the elution volumes and calibrated with a standard molecular mass kit. Protein concentration was determined according to the method of Bradford (1976), with BSA as the standard.

Circular dichroism (CD) spectrometry. The CD spectrum of the recombinant protein CM0819 was recorded at a wavelength of 200$240 \mathrm{~nm}$ stepwise $(1 \mathrm{~nm})$, with four accumulations at each step. The spectral baseline was corrected by subtracting the control. Ellipticity (mdeg) was plotted as a function of wavelength $(\mathrm{nm})$. The percentage of helicity for secondary structure determination was calculated using the K2D2 software (http://www.ogic.ca/projects/k2d2/).

\section{RESULTS}

\section{Bioinformatic analysis}

The gene $n c g l 0819$ is located at base pairs 910852-911157 on the C. glutamicum genome, and encodes a hypothetical protein of 101 amino acid residues with a theoretical molecular mass of $11.3 \mathrm{kDa}$. A BLAST search with CM0819 revealed a range of homologous ORFs that were all annotated as 'hypothetical proteins' identified in other bacterial genomes: These hypothetical proteins include cgR_0968 (locus_tag of the encoding gene; identity $100 \%$ ) of C. glutamicum strain R, CE0929 (91\%) of Corynebacterium efficiens YS-314, DIP0838 (81\%) of Corynebacterium diphtheriae NCTC 13129, CU0528 $(72 \%)$ of Corynebacterium urealyticum DSM 7109, jk1565 (68\%) of Corynebacterium jeikeium K411, MMAR_4555 (54\%) of Mycobacterium marinum M, RHA1_ro05569 (55\%) of Rhodococcus sp. RHAl, and nfa49960 (57\%) of
Nocardia farcinica IFM 10152. CM0819 also showed sequence similarity to functionally identified $\mathrm{CMs}$, the highest similarity being to the recently identified cytoplasmic MtbCM (encoded by Rv0948c, identity 57\%) (Schneider et al., 2008), and to previously identified EcCM from E. coli (27\%), MjCM from M. jannaschii (10.7\%), ScCM from S. cerevisiae (9.4\%), HpCM from $H$. polymorpha (7.9\%), and AtCM3 from A. thaliana (7.4\%). An alignment of the deduced CMs and the structurally characterized E. coli $\mathrm{CM}$ revealed a range of conserved amino acid residues (data not shown). Phylogenetic analysis based on the deduced amino acid sequences of these hypothetical proteins and the AroQ CMs characterized so far demonstrated that the functionally identified or the putative CMs from high-G + C Gram-positive bacteria clustered together, and two groups of CMs within this cluster, the mycobacteria-group CMs and the corynebacteria-group CMs, could be easily identified (Fig. 1).

\section{The mutant RES167Ancgl0819 is auxotrophic for Phe and Tyr}

In order to observe the phenotype of a $n c g l 0819$ mutant and to identify the function of $n c g l 0819$, the mutant strain RES167 $n c g l 0819$ was constructed by disruption of $n c g l 0819$ in C. glutamicum RES167 (see Methods). Phenotypic characterization indicated that mutant RES167Ancgl0819 had lost the ability to grow on minimal medium. The complementation of mutant RES167Ancgl0819 with intact $n c g l 0819$ or supplementation with three aromatic amino acids (Phe + Trp + Tyr, each $0.2 \mathrm{mM}$ ) restored its growth. Supplementation with Phe, Trp or Tyr individually, or with the combination of (Phe + Trp) or (Trp + Tyr) was not able to restore the growth of RES167Ancgl0819 in minimal medium. However, the combination of $(\mathrm{Phe}+\mathrm{Tyr}) \mathrm{did}$ restore the growth of the mutant in minimal medium (Table 2, Fig. 2). These results clearly indicated that ncgl0819 is essential for the growth of C. glutamicum in minimal medium, and suggested that this gene is involved in the biosynthesis of Phe and Tyr (but not Trp) in C. glutamicum.

\section{ncgl0819 encodes a monofunctional CM, CM0819}

The entire ncgl0819 gene was PCR amplified and cloned into E. coli BL21(DE3) via plasmid pET28-ncgl0819. Upon induction with IPTG, the E. coli BL21(DE3)/pET28ncgl0819 cells promptly synthesized new proteins that corresponded to the theoretical molecular mass of CM0819 (Fig. 3, lane 2). This protein was purified (Fig. 3, lanes 3 and 4), and its enzyme activity was determined. The enzymic assay showed that the native CM0819 had strong CM activity, but no DAHP synthase activity. Thus, it was concluded that $n c g l 0819$ encodes a monofunctional CM in C. glutamicum.

The purified CM0819 protein behaved as a single band of $\sim 12 \mathrm{kDa}$ in SDS-PAGE. When analysed by gel-filtration 


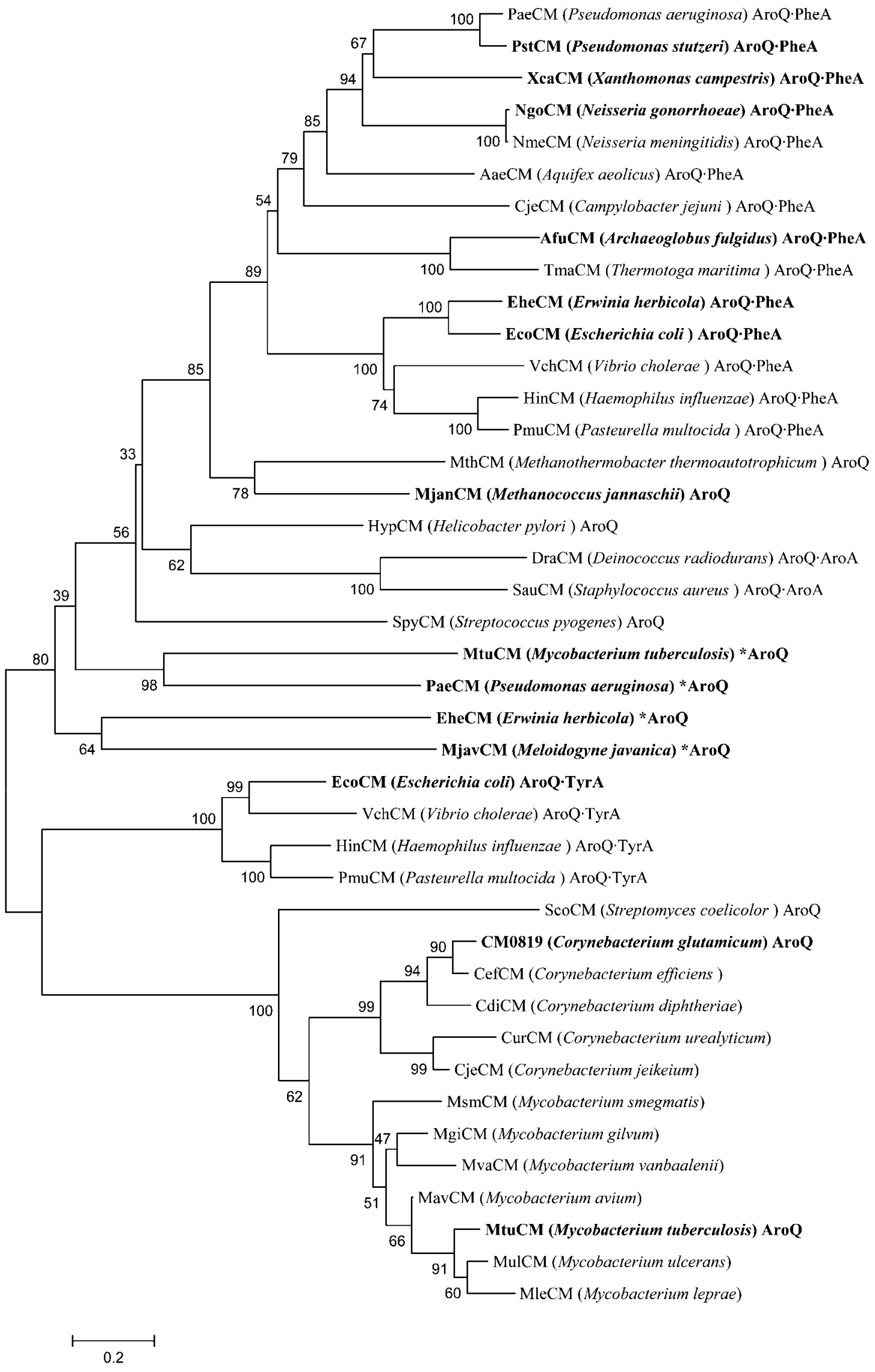


Fig. 1. Phylogenetic analysis of functionally identified (showed in bold type) and putative (annotated as hypothetical proteins) CMs. The phylogenetic tree was constructed by the neighbour-joining method with Kimura's two-parameter calculation model in MEGA version 3.1 (Kumar et al., 2004). AroQ, monofunctional CM; *AroQ, periplasmic CM. A bullet indicates a fusion joint. AroQ.PheA indicates an AroQ domain fused at its carboxyl terminus with the amino-terminal portion of a PheA domain.

chromatography, CM0819 was eluted as a molecule of $23.5 \mathrm{kDa}$. This suggested that CM0819 is a homodimer.

\section{The DAHP synthases from C. glutamicum do not show CM activity, but CM0819 stimulates DAHP synthase activity}

Previously, a two-component CM (components A and B) was reported for C. glutamicum subsp. flavum [formerly Brevibacterium flavum (Liebl 2005)]. Component A had a molecular mass of $250 \mathrm{kDa}$ and component $\mathrm{B}$ was $25 \mathrm{kDa}$. The elution patterns of component A coincided with DAHP synthase activity, and both components A and B were essential for CM activity (Shiio \& Sugimoto, 1979). In the present study, CM0819 alone showed sharp CM activity, but no DAHP synthase activity (Table 3 ). Our previous work had identified two DAHP synthases in $C$. glutamicum, encoded by ncgl0950 and ncgl2098 (Liu et al., 2008). Enzymic assays carried out in the current study showed that neither of these two DAHP synthases showed any CM activity (Table 3). However, CM0819 from C. glutamicum significantly stimulated DAHP synthase activity: DS0950 activity was increased by $39.4 \%$ and DS2098 by $253.3 \%$. Stimulation of CM activity by either of the two DAHP synthases was not observed (Table 3 ).

\section{CM0819 and DS2098 interact with each other}

The fact that CM0819 stimulated DS2098 DAHP synthase activity suggested a possible interaction between these two proteins. Our results from affinity chromatography confirmed this (Fig. 4): pre-loading of CM0819 onto the HisBind protein purification column resulted in subsequent binding of DS2098 (which did not have a His-tag and thus did not bind on itself) onto the column. This suggested that CM0819 and DS2098 might form a complex and that their function might be regulated.

It was previously reported that $B$. subtilis had a bifunctional DAHP synthase-CM and that the DAHP synthase activity was subject to feedback inhibition by chorismate (Jensen \&
Nester, 1965). We examined the effects of chorismate and prephenate on the two DAHP synthases (DS2098 and DS0950) from C. glutamicum. The results indicated that DS2098 activity was not affected by chorismate or prephenate when DS2098 was alone. However, in the presence of CM0819, the DAHP synthase activity of DS2098 was inhibited by chorismate (at $1 \mathrm{mM}$ ) by $45.7 \%$ and by prephenate by $24.9 \%$ (at $1 \mathrm{mM}$ ) and $41.0 \%$ (at $2 \mathrm{mM}$ ). In contrast to DS2098, DS0950 activity was inhibited by chorismate (at $1 \mathrm{mM}$ ) by $36.1 \%$ and by prephenate (at $1 \mathrm{mM}$ ) by $17.3 \%$, when it was alone. When DS0950 and CM0819 were together in the mixture, the DAHP synthase activity of DS0950 was inhibited by chorismate (at $1 \mathrm{mM}$ ) by $43.2 \%$ and by prephenate (at $1 \mathrm{mM}$ ) by $18.1 \%$, similar to the inhibition seen when DS0950 was alone.

\section{CM0819 activity is not regulated by Phe, Tyr and Trp, but is affected by metal ions}

The feedback regulation effect of Phe, Tyr and Trp on CM0819 activity was determined for each individual amino acid. There was no observable feedback regulation of $\mathrm{CM}$ activity at concentrations of $0.01-10 \mathrm{mM}$ of any of Phe, Trp or Tyr. This is similar to MtbCM from Mycobacterium tuberculosis, which was not subject to feedback regulation by Phe, Trp or Tyr (Kim et al., 2006).

Effects of various divalent metal ions on CM0819 activity were studied. $\mathrm{Mg}^{2+}$ and EDTA at concentrations of 0.1$2 \mathrm{mM}$ did not affect CM0819 activity. $\mathrm{Mn}^{2+}, \mathrm{Co}^{2+}$ and $\mathrm{Ni}^{2+}$, each at $2 \mathrm{mM}$, decreased CM0819 activity by $38.9 \%$, $52.4 \%$ and $41.8 \%$, respectively.

The kinetic constants of the CM0819 were determined. The $K_{\mathrm{m}}$ for chorismate was determined to be $2.83 \pm 0.53 \mathrm{mM}$, and $V_{\max }$ was $2.75 \pm 0.52 \mu \mathrm{mol} \mathrm{min}{ }^{-1} \mathrm{mg}^{-1}$. The molar catalytic activity $\left(k_{\text {cat }}\right)$ was calculated to be $1.05 \pm 0.19 \mathrm{~s}^{-1}$.

\section{CM0819 is an $\alpha$-helix-predominant protein}

The CD spectrum of the CM0819 was recorded on a JASON spectropolarimeter (Fig. 5). The data were analysed

Table 2. Phenotypes of mutant RES167 $\mathrm{n}$ cgl0819 grown in minimal medium with addition of different aromatic amino acids (each at final concentration of $0.2 \mathrm{mM}$ )

\begin{tabular}{|c|c|c|c|c|c|c|c|c|}
\hline & None & Phe & Tyr & Trp & Phe + Tyr & Phe + Trp & $\operatorname{Tyr}+\operatorname{Trp}$ & $\begin{array}{c}\text { Phe+ } \\
\text { Tyr + Trp }\end{array}$ \\
\hline RES167 & + & + & + & + & + & + & + & + \\
\hline 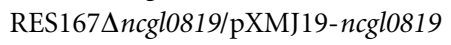 & + & + & + & + & + & + & + & + \\
\hline
\end{tabular}




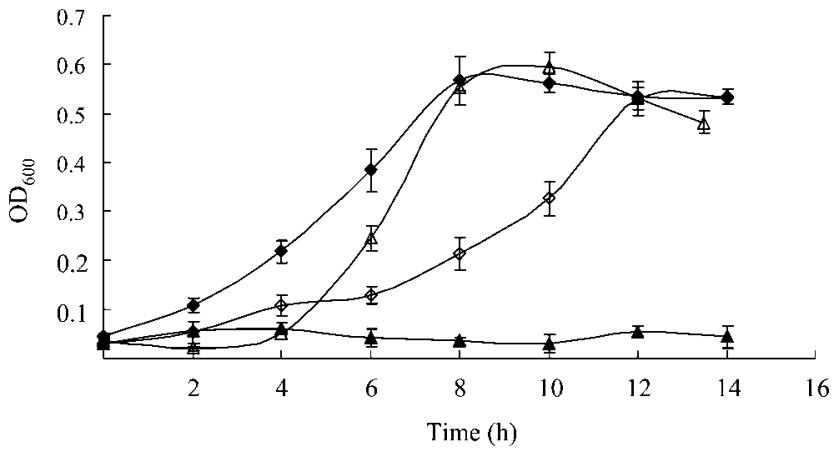

Fig. 2. Growth curves of C. glutamicum strains. To observe the phenotype after gene disruption or complementation, stains were cultivated in minimal medium with $2 \mathrm{mM}$ glucose as the only carbon and energy source. $\downarrow$, RES167; $\Delta$, RES1674ncg/0819; $\triangle$, RES167Ancgl0819 complemented with ncgl0819; $\diamond$, RES167 $\Delta$ cgl0819 in minimal medium with Phe and Tyr (each $0.2 \mathrm{mM})$. Data are means $\pm S D$ from three parallel cultures.

using the K2D2 software, and the results revealed that CM0819 had $67.5 \% \alpha$-helices and $3.2 \% \beta$-sheets, indicating a predominantly helical structure for the enzyme. Similarly, the CMs from yeast and E. coli were reported also to be helix-predominant proteins (Lee et al., 1995; Strater et al., 1997).

\section{DISCUSSION}

In this study, the gene (ncgl0819) encoding chorismate mutase (CM0819) from C. glutamicum was identified and the CM0819 enzyme was characterized. To our knowledge, this is the first functionally identified CM gene of the genus Corynebacterium. Highly conserved hypothetical proteins

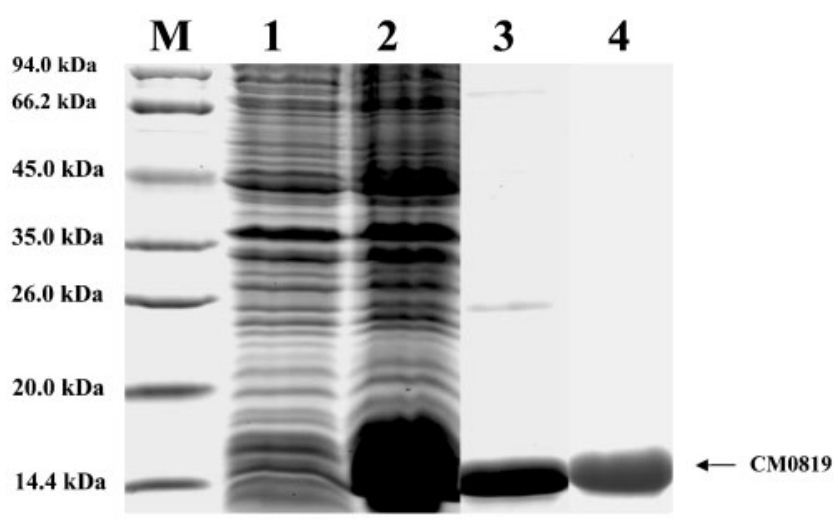

Fig. 3. Cloning and expression of CM0819 in E. coli. Lanes: M, molecular mass markers; 1, E. coli BL21(DE3)/pET28-ncg/0819, not induced by IPTG; 2, E. coli BL21(DE3)/pET28-ncg/0819, induced by IPTG; 3, purification of CM0819, after His-tag chromatography; 4 , purification of $\mathrm{CM} 0819$, after gel filtration.
Table 3. Determination of $\mathrm{CM}$ activity and interactions between CM0819 and DAHP synthases from C. glutamicum RES167

DAHP synthases are encoded by $n c g l 0950$ and ncgl2098 (Liu et al., 2008). Data are means \pm SD from three parallel determinations.

\begin{tabular}{|lcc|}
\hline & $\begin{array}{c}\text { Relative CM } \\
\text { activity (\%) }\end{array}$ & $\begin{array}{c}\text { Relative DAHP } \\
\text { synthase activity (\%) }\end{array}$ \\
\hline CM0819 & $100^{\star} \pm 9.0$ & None \\
DS0950 & None & $100 \dagger \pm 12.2$ \\
DS0950+CM0819 & $102.1 \pm 7.2$ & $139.4 \pm 25.9$ \\
DS2098 & None & $100 \ddagger \pm 35.3$ \\
DS2098+CM0819 & $105.9 \pm 7.0$ & $353.3 \pm 33.3$ \\
\hline
\end{tabular}

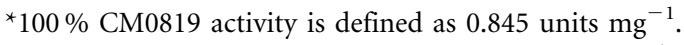
$\dagger 100 \%$ DS0950 activity is defined as 0.034 units $\mathrm{mg}^{-1}$.

$\ddagger 100 \%$ DS2098 activity is defined as 0.032 units $\mathrm{mg}^{-1}$. Purified enzymes were used in the assay.

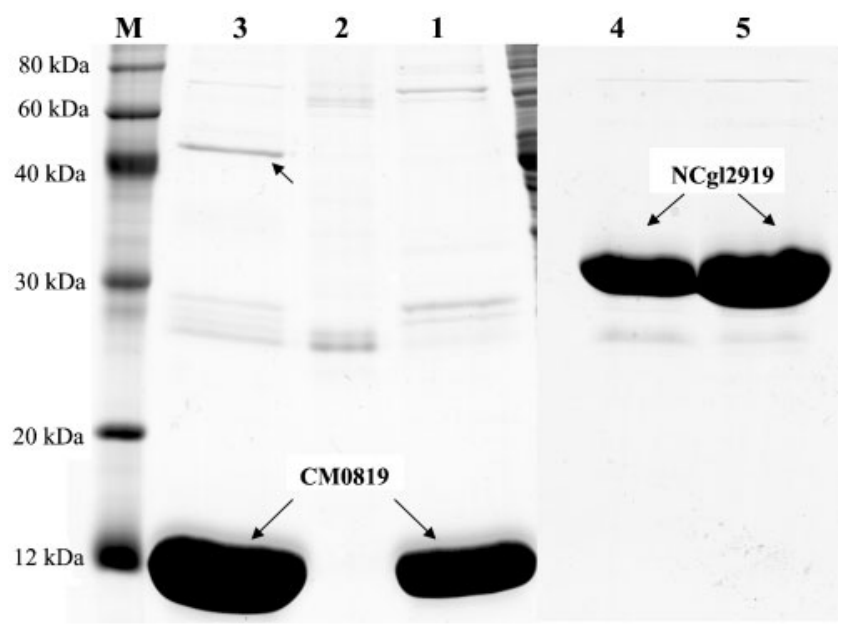

Fig. 4. SDS-PAGE of proteins eluted from columns of His-tag chromatography, showing the interaction between CM0819 and DS2098. M, molecular mass markers. 1, His-tag labelled CM0819, eluted from a His-tag binding chromatography column loaded with BL21(DE3)/pET28-ncg/0819 cellular lysate. 2, Eluate from column loaded with BL21(DE3)/pET21-ncg/2098 cellular lysate (containing DS2098 without His-tag), indicating no binding of DS2098. 3, Interaction of CM0819 with DS2098. Both proteins were simultaneously eluted from a His-tag binding chromatography column, which was first loaded with BL21(DE3)/pET28ncgl0819 cellular lysate and then with BL21(DE3)/pET21ncgl2098 cellular lysate. 4, His-tagged NCgl2919 (fumarylpyruvate hydrolase), eluted from a His-tag binding chromatography column loaded with BL21(DE3)/pET28-ncg/2919 cellular lysate. 5 , A His-tag binding chromatography column was first loaded with BL21(DE3)/pET28-ncg/2919 cellular lysate and then loaded with BL21(DE3)/pET21-ncg/2098 cellular lysate. Only NCgl2919 was eluted, indicating that NCgl2919 did not interact with DS2098. 


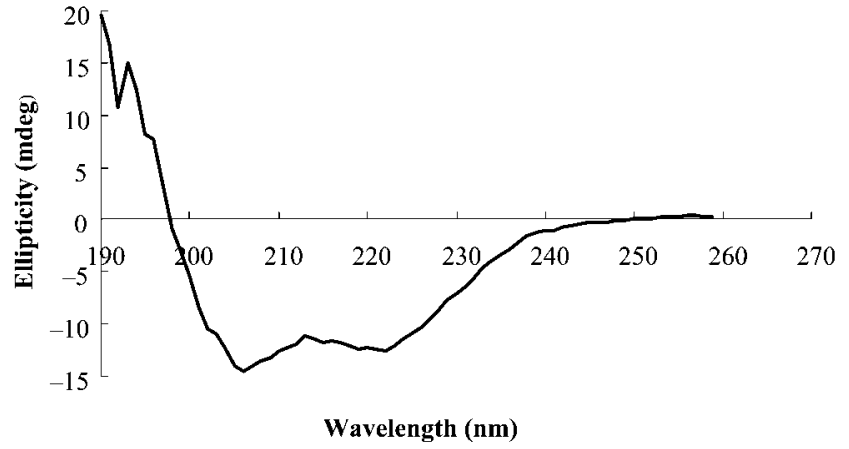

Fig. 5. CD spectrum of CM0819, suggesting a predominantly $\alpha$ helical secondary structure of the protein. The percentage of helicity for secondary-structure determination was calculated using the K2D2 software. The spectrum confirms a highly helical structure of the recombinant protein with signature peaks at 207 and $222 \mathrm{~nm}$.

homologous to CM0819 were identified in all currently known Corynebacterium genomes. We deduced that those hypothetical proteins would function as $\mathrm{CMs}$ and are involved in the central shikimate pathway in Corynebacterium species. According to the sequencesimilarity analysis, CM0819 is more closely related to the cytoplasmic MtbCM (Rv0948c) than to the secreted MtbCM (Rv1885c) in Mycobacterium tuberculosis. Considering that $n c g l 0819$ was necessary for growth of $C$. glutamicum in minimal medium, we conclude that ncgl0819 is the sole CM gene in C. glutamicum.

Our results indicate that CM0819 is a homodimer, and its secondary structure is rich in $\alpha$-helices. Thus, it is proposed that CM0819 is a new member of the monofunctional AroQ class of CMs. Further, this CM0819 stimulates DAHP synthase activity in C. glutamicum, a new observation that has not been reported before. It is proposed that CM0819 interacts with DS2098 and the two proteins possibly form a complex. This hypothesis is supported by the previous observation that CM and DAHP synthase activities associated with each other and were isolated as a protein complex from C. glutamicum (Sugimoto \& Shiio, 1980b).

CM catalyses the committed step in Phe and Tyr biosynthesis (Prakash et al., 2005). The CMs of the AroQ class originating from various hosts are subject to different types of regulation. EcCM exists as a fusion protein in $E$. coli and is subject to allosteric inhibition only by pathwayspecific inhibitors (Prakash et al., 2005). The ScCM of $S$. cerevisiae and AnCM of Aspergillus nidulans are inhibited by Tyr and Phe and are stimulated by Trp (Krappmann et al., 1999; Schmidheini et al., 1989). Similar to MtbCM (Kim et al., 2006), CM0819 in C. glutamicum is not subject to any regulation by aromatic amino acids. But CM0819 together with chorismate and prephenate regulates DS2098 activity via allosteric inhibition, similar to what was observed for the bifunctional CM from B. subtilis (Jensen \& Nester, 1965). However, CM0819 and DS2098 from C. glutamicum differ from the bifunctional $\mathrm{CM}$ from $B$. subtilis in that the former was more sensitive to chorismate regulation (this study) and the latter was more sensitive to prephenate regulation (Jensen \& Nester, 1965). Previously, Gosset et al. (2001) reported that DAHP synthase from Xanthomonas campestris was subject to allosteric inhibition by chorismate and was slightly inhibited by Trp. We found that DS2098 alone was not subject to inhibition by chorismate (this study) and was slightly inhibited by Trp (Liu et al., 2008). When DS2098 and CM0819 occurred together, allosteric inhibition of the DAHP synthase activity was observed, a new observation that has not been reported before.

\section{ACKNOWLEDGEMENTS}

The authors are grateful to $\mathrm{Dr}$ De-Feng $\mathrm{Li}$ at the Institute of Biophysics, Chinese Academy of Sciences (Beijing) for his assistance in determination of the CM0819 CD spectrum. This work was supported by a grant from the Ministry of Science and Technology of China (863-project, Grant No. 2006AA02Z219) and the National Nature Science Foundation of China (30725001).

\section{REFERENCES}

Ball, S. G., Wickner, R. B., Cottarel, G., Schaus, M. \& Tirtiaux, C. (1986). Molecular cloning and characterization of ARO7-OSM2, a single yeast gene necessary for chorismate mutase activity and growth in hypertonic medium. Mol Gen Genet 205, 326-330.

Bradford, M. M. (1976). A rapid and sensitive method for the quantitation of microgram quantities of protein utilizing the principle of protein-dye binding. Anal Biochem 72, 248-254.

Calhoun, D. H., Bonner, C. A., Gu, W., Xie, G. \& Jensen, R. A. (2001). The emerging periplasm-localized subclass of AroQ chorismate mutases, exemplified by those from Salmonella typhimurium and Pseudomonas aeruginosa. Genome Biol 2, RESEARCH0030.

Chavez-Bejar, M. I., Lara, A. R., Lopez, H., Hernandez-Chavez, G., Martinez, A., Ramirez, O. T., Bolivar, F. \& Gosset, G. (2008). Metabolic engineering of Escherichia coli for L-tyrosine production by expression of genes coding for the chorismate mutase domain of the native chorismate mutase-prephenate dehydratase and a cyclohexadienyl dehydrogenase from Zymomonas mobilis. Appl Environ Microbiol 74, 3284-3290.

Chook, Y. M., Ke, H. \& Lipscomb, W. N. (1993). Crystal structures of the monofunctional chorismate mutase from Bacillus subtilis and its complex with a transition state analog. Proc Natl Acad Sci U S A 90, 8600-8603.

Chook, Y. M., Gray, J. V., Ke, H. \& Lipscomb, W. N. (1994). The monofunctional chorismate mutase from Bacillus subtilis. Structure determination of chorismate mutase and its complexes with a transition state analog and prephenate, and implications for the mechanism of the enzymatic reaction. J Mol Biol 240, 476-500.

Davidson, B. E. \& Hudson, G. S. (1987). Chorismate mutaseprephenate dehydrogenase from Escherichia coli. Methods Enzymol 142, 440-450.

Eberhard, J., Raesecke, H. R., Schmid, J. \& Amrhein, N. (1993). Cloning and expression in yeast of a higher plant chorismate mutase. Molecular cloning, sequencing of the cDNA and characterization of 
the Arabidopsis thaliana enzyme expressed in yeast. FEBS Lett 334, 233-236.

Gosset, G., Bonner, C. A. \& Jensen, R. A. (2001). Microbial origin of plant-type 2-keto-3-deoxy-D-arabino-heptulosonate 7-phosphate synthases, exemplified by the chorismate- and tryptophan-regulated enzyme from Xanthomonas campestris. J Bacteriol 183, 4061-4070.

Gray, J. V., Golinelli-Pimpaneau, B. \& Knowles, J. R. (1990). Monofunctional chorismate mutase from Bacillus subtilis: purification of the protein, molecular cloning of the gene, and overexpression of the gene product in Escherichia coli. Biochemistry 29, 376-383.

Helmstaedt, K., Krappmann, S. \& Braus, G. H. (2001). Allosteric regulation of catalytic activity: Escherichia coli aspartate transcarbamoylase versus yeast chorismate mutase. Microbiol Mol Biol Rev 65, 404-421.

Helmstaedt, K., Heinrich, G., Merkl, R. \& Braus, G. H. (2004). Chorismate mutase of Thermus thermophilus is a monofunctional AroH class enzyme inhibited by tyrosine. Arch Microbiol 181, 195-203.

Horton, R. M., Hunt, H. D., Ho, S. N., Pullen, J. K. \& Pease, L. R. (1989). Engineering hybrid genes without the use of restriction enzymes: gene splicing by overlap extension. Gene 77, 61-68.

Hudson, G. S., Wong, V. \& Davidson, B. E. (1984). Chorismate mutase/prephenate dehydrogenase from Escherichia coli K12: purification, characterization, and identification of a reactive cysteine. Biochemistry 23, 6240-6249.

Ikeda, M. (2005). L-Tryptophan production. In Handbook of Corynebacterium glutamicum, pp. 489-51. Edited by L. Eggeling \& M. Bott. Boca Raton, FL: CRC Press.

Ikeda, M. (2006). Towards bacterial strains overproducing Ltryptophan and other aromatics by metabolic engineering. Appl Microbiol Biotechnol 69, 615-626.

Ikeda, M. \& Nakagawa, S. (2003). The Corynebacterium glutamicum genome: features and impacts on biotechnological processes. Appl Microbiol Biotechnol 62, 99-109.

Jakoby, M., Ngougto-Nkili, C.-E. \& Burkovski, A. (1999). Construction and application of new Corynebacterium glutamicum vectors. Biotechnol Tech 13, 437-441.

Jensen, R. A. \& Nester, E. W. (1965). The regulatory significance of intermediary metabolites: control of aromatic acid biosynthesis by feedback inhibition in Bacillus subtilis. J Mol Biol 12, 468-481.

Kim, S. K., Reddy, S. K., Nelson, B. C., Vasquez, G. B., Davis, A., Howard, A. J., Patterson, S., Gilliland, G. L., Ladner, J. E. \& Reddy, P. T. (2006). Biochemical and structural characterization of the secreted chorismate mutase (Rv1885c) from Mycobacterium tuberculosis H37Rv: an ${ }^{*}$ AroQ enzyme not regulated by the aromatic amino acids. $J$ Bacteriol 188, 8638-8648.

Konopka, A. (1993). Isolation and characterization of subsurface bacterium that degrades aniline and methylanilines. FEMS Microbiol Lett 111, 93-100.

Krappmann, S., Helmstaedt, K., Gerstberger, T., Eckert, S., Hoffmann, B., Hoppert, M., Schnappauf, G. \& Braus, G. H. (1999). The aroC gene of Aspergillus nidulans codes for a monofunctional, allosterically regulated chorismate mutase. J Biol Chem 274, 2227522282.

Krappmann, S., Pries, R., Gellissen, G., Hiller, M. \& Braus, G. H. (2000). HARO7 encodes chorismate mutase of the methylotrophic yeast Hansenula polymorpha and is derepressed upon methanol utilization. J Bacteriol 182, 4188-4197.

Kumar, S., Tamura, K. \& Nei, M. (2004). MEGA3: integrated software for molecular evolutionary genetics analysis and sequence alignment. Brief Bioinform 5, 150-163.
Lee, A. Y., Karplus, P. A., Ganem, B. \& Vlardy, J. (1995). Atomic structure of the buried catalytic pocket of Escherichia coli chorismate mutase. J Am Chem Soc 117, 3627-3628.

Liebl, W. (2005). Corynebacterium taxonomy. In Handbook of Corynebacterium glutamicum, pp. 9-36. Edited by L. Eggeling \& M. Bott. Boca Raton, FL: CRC Press.

Liu, Y. J., Li, P. P., Zhao, K. X., Wang, B. J., Jiang, C. Y., Drake, H. L. \& Liu, S. J. (2008). Corynebacterium glutamicum contains 3-deoxy-Darabino-heptulosonate 7-phosphate synthases that display novel biochemical features. Appl Environ Microbiol 74, 5497-5503.

MacBeath, G., Kast, P. \& Hilvert, D. (1998). A small, thermostable, and monofunctional chorismate mutase from the archaeon Methanococcus jannaschii. Biochemistry 37, 10062-10073.

Prakash, P., Aruna, B., Sardesai, A. A. \& Hasnain, S. E. (2005). Purified recombinant hypothetical protein coded by open reading frame Rv1885c of Mycobacterium tuberculosis exhibits a monofunctional AroQ class of periplasmic chorismate mutase activity. J Biol Chem 280, 19641-19648.

Qamra, R., Prakash, P., Aruna, B., Hasnain, S. E. \& Mande, S. C. (2006). The $2.15 \AA$ crystal structure of Mycobacterium tuberculosis chorismate mutase reveals an unexpected gene duplication and suggests a role in host-pathogen interactions. Biochemistry 45, 69977005.

Sambrook, J., Fritsch, E. F. \& Maniatis, T. (1989). Molecular Cloning: a Laboratory Manual, 2nd edn. Cold Spring Harbor, NY: Cold Sping Harbor Laboratory.

Schafer, A., Tauch, A., Jager, W., Kalinowski, J., Thierbach, G. \& Puhler, A. (1994). Small mobilizable multi-purpose cloning vectors derived from the Escherichia coli plasmids pK18 and pK19: selection of defined deletions in the chromosome of Corynebacterium glutamicum. Gene 145, 69-73.

Schmidheini, T., Sperisen, P., Paravicini, G., Hutter, R. \& Braus, G. (1989). A single point mutation results in a constitutively activated and feedback-resistant chorismate mutase of Saccharomyces cerevisiae. J Bacteriol 171, 1245-1253.

Schneider, C. Z., Parish, T., Basso, L. A. \& Santos, D. S. (2008). The two chorismate mutases from both Mycobacterium tuberculosis and Mycobacterium smegmatis: biochemical analysis and limited regulation of promoter activity by aromatic amino acids. J Bacteriol 190, 122-134.

Schoner, R. \& Herrmann, K. M. (1976). 3-Deoxy-D-arabinoheptulosonate 7-phosphate synthase. Purification, properties, and kinetics of the tyrosine-sensitive isoenzyme from Escherichia coli. J Biol Chem 251, 5440-5447.

Shen, X. H., Jiang, C. Y., Huang, Y., Liu, Z. P. \& Liu, S. J. (2005). Functional identification of novel genes involved in the glutathioneindependent gentisate pathway in Corynebacterium glutamicum. Appl Environ Microbiol 71, 3442-3452.

Shiio, I. \& Sugimoto, S. (1979). Two components of chorismate mutase in Brevibacterium flavum. J Biochem 86, 17-25.

Siehl, D. L. (1997). Inhibitors of EPSP synthase, glutamine synthetase and histidine synthesis. In Herbicide Activity: Toxicology, Biochemistry and Molecular Biology, pp. 37-67. Edited by R. M. Roe, J. D. Burton \& R. J. Kurh. Amsterdam/Oxford: IOS.

Strater, N., Schnappauf, G., Braus, G. \& Lipscomb, W. N. (1997). Mechanisms of catalysis and allosteric regulation of yeast chorismate mutase from crystal structures. Structure 5, 1437-1452.

Sugimoto, S. \& Shiio, I. (1980a). Purification and properties of dissociable chorismate mutase from Brevibacterium flavum. J Biochem 88, 167-176.

Sugimoto, S. \& Shiio, I. (1980b). Purification and properties of bifunctional 3-deoxy-D-arabino-heptulosonate 7-phosphate synthe- 
tase-chorismate mutase component A from Brevibacterium flavum. J Biochem 87, 881-890.

Tauch, A., Kassing, F., Kalinowski, J. \& Puhler, A. (1995). The Corynebacterium xerosis composite transposon Tn5432 consists of two identical insertion sequences, designated IS1249, flanking the erythromycin resistance gene ermCX. Plasmid 34, 119-131.

Tauch, A., Kirchner, O., Loffler, B., Gotker, S., Puhler, A. \&

Kalinowski, J. (2002). Efficient electrotransformation of Coryne- bacterium diphtheriae with a mini-replicon derived from the Corynebacterium glutamicum plasmid pGA1. Curr Microbiol 45, 362-367.

Yakandawala, N., Romeo, T., Friesen, A. D. \& Madhyastha, S. (2008). Metabolic engineering of Escherichia coli to enhance phenylalanine production. Appl Microbiol Biotechnol 78, 283-291.

Edited by: L. Heide 